\title{
DESIGN OF AN ELEMENTAL ANALYSIS SYSTEM FOR CELSS RESEARCH
}

\author{
Steven H. Schwartzkopf
}

Plant Growth Laboratory. University of California, Davis, Davis. California 95616 (USA)

\begin{abstract}
The results of experiments conducted with higher plants in tightly sealed growth chambers provide definite evidence that the physical closure of a chamber has significant effects on many aspects of a plant's biology. One of these effects is seen in the change in rates of uptake. distribution, and re-release of nutrient elements by the plant (mass balance). Experimental data indicates that these rates are different from those recorded for plants grown in open field agriculture, or in open growth chambers. Since higher plants are a crucial component of a CELSS. it is important that the consequences of these rate differences be understood with regard to the growth and yield of the plants. This paper will focus on the description of a system for elemental analysis which can be used to monitor the mass balance of nutrient elements in CELSS experiments. Additionally, data on the uptake of nutrient elements by higher plants grown in a growth chamber will be presented.
\end{abstract}

\section{INTRODUCTION}

As Carl Sprengel pointed out over 100 years ago. the productivity of a plant can be inhibited by the shortage of a single nutrient element ("Liebig's" Law of the Minimum). even when all the other elements are in ample supply. In CELSS applications. where high plant productivity is desirable, it is necessary to monitor and maintain the concentrations of all the nutrient elements to prevent this limitation from occurring. Additionally. because plants may take up more than they require of elements that are in high concentrations (i.e. luxury consumption). with potential effects on nutritional quality, it is important to ensure that excessively high concentrations are not reached. For this presentation, we will concentrate on describing an elemental analysis system developed for CELSS research use.

\section{METHODS}

Initially. we evaluated four different methods for the monitoring of nutrient elements in hydroponic/aeroponic plant growth systems. These included atomic absorption/flame photometry (AA/FP). inductively coupled plasma (ICP). continuous flow analysis (CFA) and ion chromatography (IC).

The advantages of IC over the other monitoring techniques include:

1) Low purchase price: Approximately $\$ 25,000$ for an anion/cation system.

2) Low operating costs: Reagents are inexpensive, and the only elements that require replacement in general use are the columns.

3) Very easy to operate: No extensive training is required to run the IC.

4) Small sample size: Only about $0.1 \mathrm{ml}$ sample is required for analysis.

5) May be automated: Auto-samplers are available now, but if a pressurized nutrient line is available. all that is required is to add a solenoid valve between the IC and the nutrient line.

The first step in development of the nutrient analysis system was characterization of the IC. Figure 1 illustrates a typical chromatogram. Preliminary analysis indicated that using peak height as an indicator of ionic concentration was equal to or better than using integrated peak area. Because peak height is easier to measure and significantly less complicated to obtain via a computerized data acquisition system, we chose to use peak 


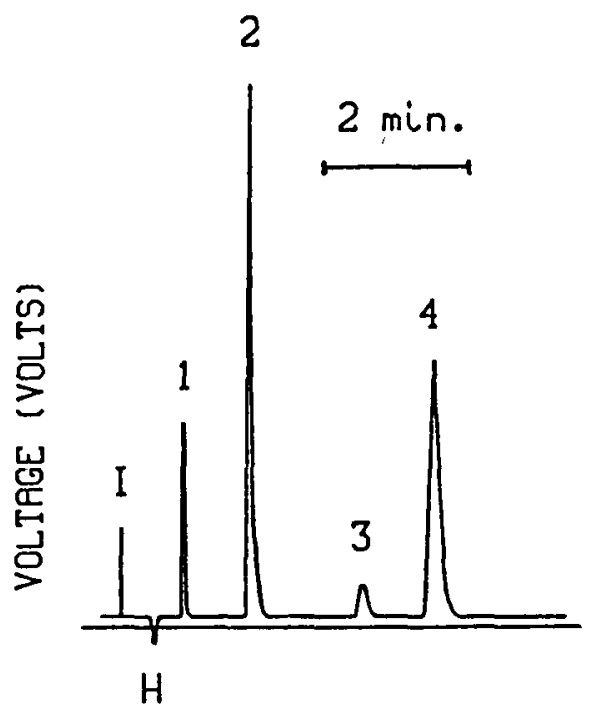

Fig. 1. Typical ion chromatogram. I, injection mark: H, water "peak": 1 , chloride: 2 , nitrate: 3. phosphate: 4 , sulfate.

height for ionic concentration measurements. Standard solutions were mixed, and standard curves of concentration versus detector voltage were constructed. These standard curves were then used to determine the ionic concentrations in nutrient solution samples collected during a plant growth experiment.

The data presented below was collected during an experiment in which plant growth and uptake of anions from the nutrient solution were monitored. A first crop of lettuce plants were grown in modified, half-strength Hoaglands' solution. For the experiment described below. a second crop of lettuce plants was then grown in the same solution. but supplemented with a solution obtained from wet-oxidation of the lettuce heads from the first crop. This group was compared with a control group grown in a fresh batch of half-strength modified Hoaglands' solution.

The nutriculture system used in the experiment described here is housed in a $3 \mathrm{~m}^{2}$ controlled environment room, and has been described by Schwartzkopf, et al. 1986 . Temperature was $25 / 20 \mathrm{C}$ day/night. relative humidity was $70 \%$. light averaged $600 \mu \mathrm{mol} / \mathrm{m}^{2} / \mathrm{s}$, nutrient solution temperature was $23 \mathrm{C}$. pH was 6.4 , and atmospheric $\mathrm{CO}_{2}$ was kept at ambient concentration.

\section{RESULTS}

Figure 2 is a composite of the standard curves constructed with the IC. lonic solution concentrations ranged from full strength, modified Hoaglands' to pure, deionized water. As this figure indicates, the IC is a very precise instrument. Standard deviations ranged from 1 to $5 \%$ of the value of the mean for each of the four anions.

Figure 3 is a composite showing the concentrations of the anionic species during the course of the experiment. This graph shows the accumulation of chloride in the control and wet-ox solutions. The accumulation of chloride was due to the addition of $0.1 \mathrm{M} \mathrm{HCl}$ to maintain $\mathrm{pH}$. The apparent increase in nitrate, sulfate and phosphate during the first two weeks was due to a malfunction in the automated makeup water system. which allowed the nutrient solution volume to decrease by about $20 \%$. thus producing an apparent increase in the concentrations of these three anions. Nitrate uptake was slightly faster in the wet-ox grown plants than in the control plants. Coupled with the lower initial nitrate concentration. this reduced the nitrate level in the wet-ox solution to a barely measurable concentration by the 24 th day of the experiment. Phosphate concentrations followed nearly the same pattern in both groups, and final concentrations were equal. despite the fact that the overall concentration of phosphate in the wet-ox solution was about half that of the control. Sulfate, on the other hand. not only followed the same general course of the control. but was equivalent in concentrations as well.

Finally, upon harvesting the lettuce. no statistically significant differences were found between the dry weights of the two groups of plants (Table 1). A blind taste test was conducted with this lettuce, and 40 volunteer subjects found the wet-ox grown lettuce too bitter to eat! We are beginning tissue analysis to determine what caused this bitterness. but it may be due to a nutrient limitation during the last few days of growth. possibly nitrate. as shown in Figure 3 (W. Berry. personal communication). This result emphasizes both the need for nutrient element analysis and the control of mass balance. in order to preserve the nutritional quality of foodstuffs grown in a CELSS. 


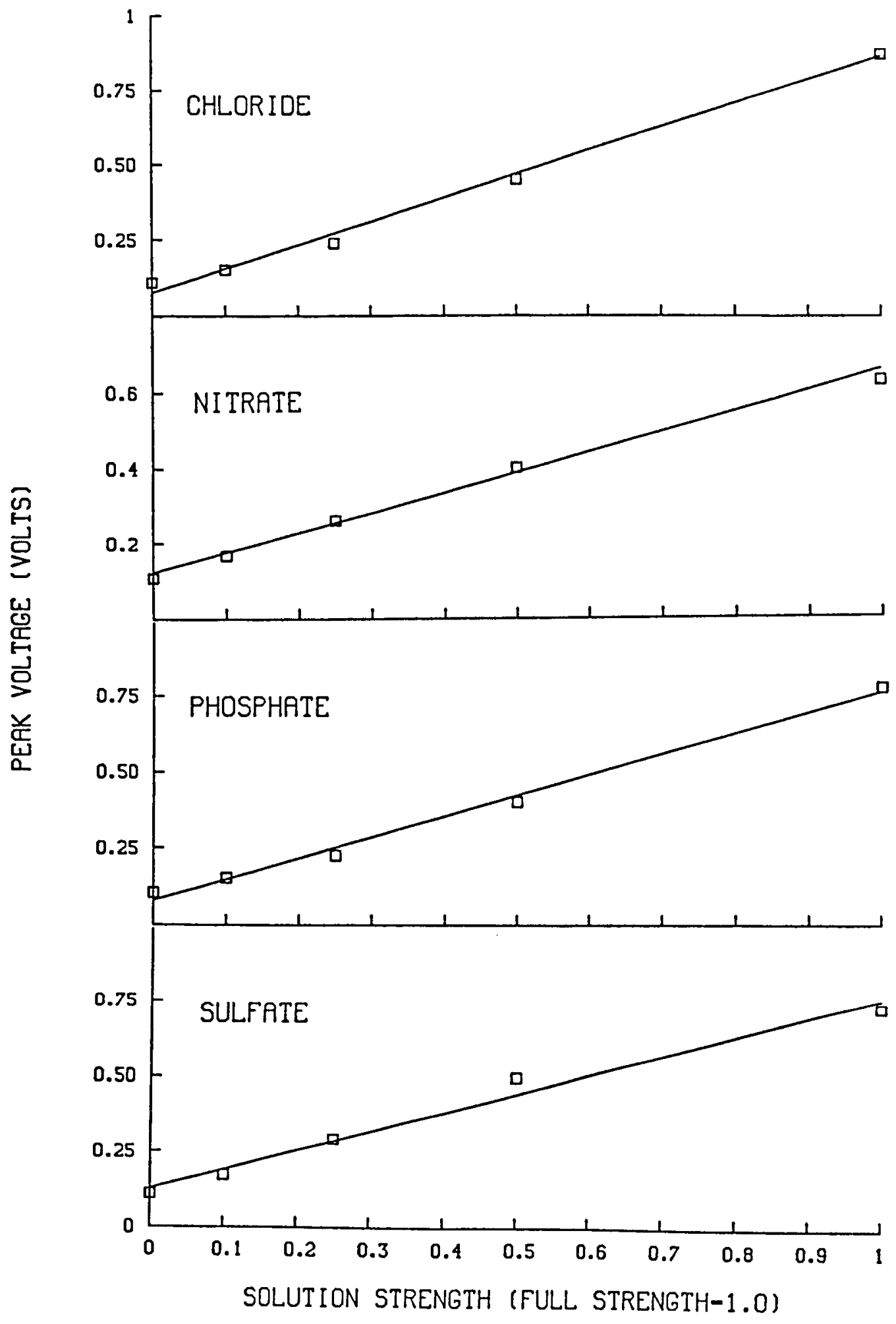

Fig. 2. Standard curves for anions. 


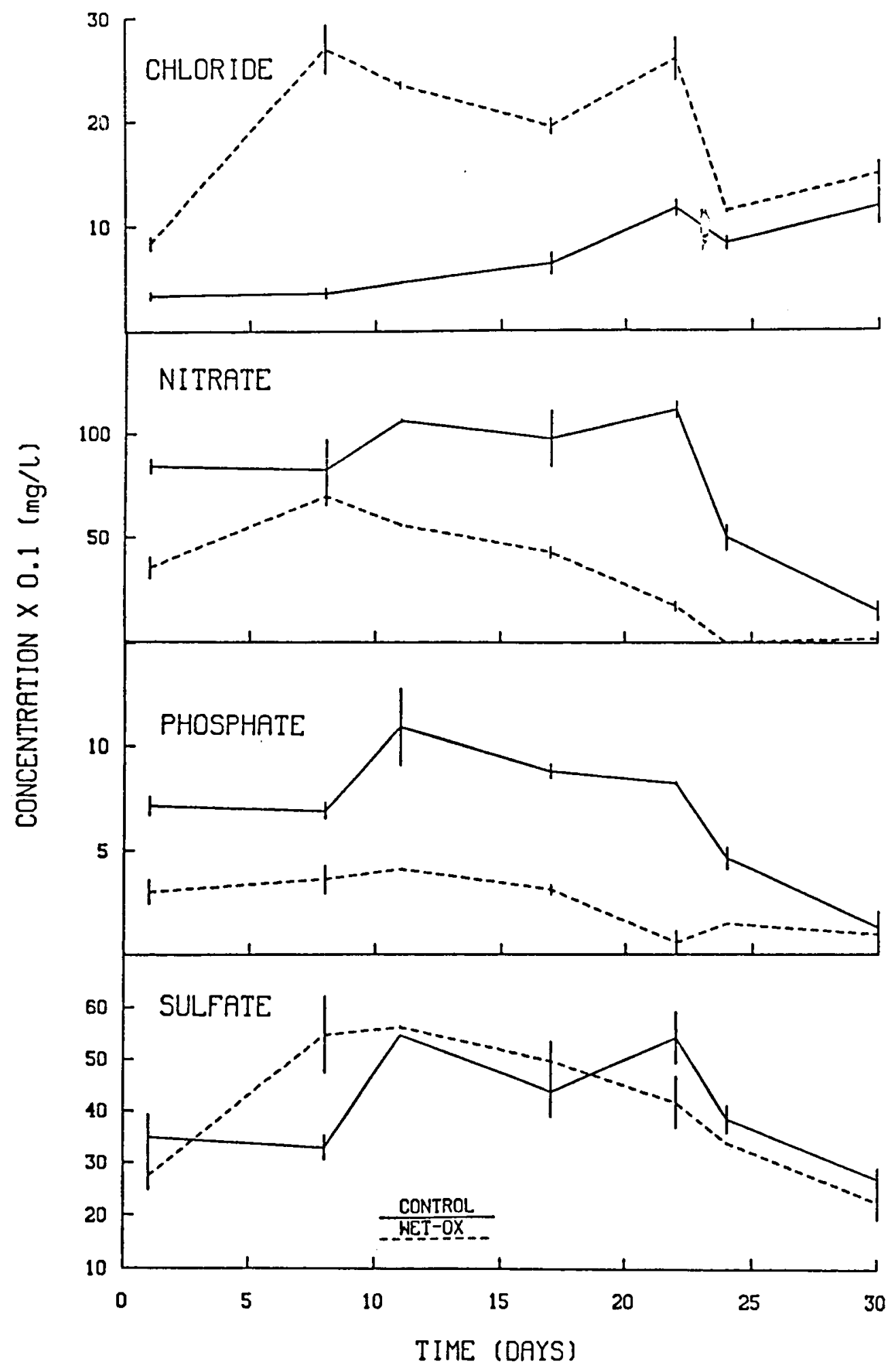

Fig. 3. Anion concentrations during wet oxidation experiment. 
TABLE 1 Dry Weights of Tops and Roots of 5 Week Old Lettuce Plants

\begin{tabular}{llll}
\hline & & Wet-Ox & Control \\
\hline Top & $\bar{x}$ & 2.80 & 2.27 \\
& $s$ & 0.80 & 0.63 \\
Root & $\bar{x}$ & 0.39 & 0.48 \\
& $s$ & 0.078 & 0.093 \\
\hline
\end{tabular}

\section{CONCLUSION}

The use of ion chromatography for analysis of nutrient solution ion concentrations is an accurate. relatively rapid monitoring method. Initial evaluation indicates that the technique is well suited to the analysis of nutrient solution samples from hydroponic plant growth experiments for CELSS applications.

\section{REFERENCES}

Schwartzkopf. S.H.. D. Dudzinski, and S. Minners. 1986. The Effects of Nutrient Solution Sterilization on the Growth and Yield of Hydroponically-Grown Lettuce. Accepted for Publication in HortScience. 